\title{
Wall Clutter Mitigation and Target Detection Using Discrete Prolate Spheroidal Sequences
}

\author{
Zhihui Zhu and Michael B. Wakin \\ Department of Electrical Engineering and Computer Science \\ Colorado School of Mines, Golden, CO, 80401, USA \\ \{zzhu,mwakin\}@mines.edu
}

\begin{abstract}
We present a new method for mitigating wall return and a new greedy algorithm for detecting stationary targets after wall clutter has been cancelled. Given limited measurements of a stepped-frequency radar signal consisting of both wall and target return, our objective is to detect and localize the potential targets. Modulated Discrete Prolate Spheroidal Sequences (DPSS's) form an efficient basis for sampled bandpass signals. We mitigate the wall clutter efficiently within the compressive measurements through the use of a bandpass modulated DPSS basis. Then, in each step of an iterative algorithm for detecting the target positions, we use a modulated DPSS basis to cancel nearly all of the target return corresponding to previously selected targets. With this basis, we improve upon the target detection sensitivity of a Fourier-based technique.
\end{abstract}

Keywords-radar imaging, wall clutter mitigation, target detection, Compressive Sensing, Discrete Prolate Spheroidal Sequences

\section{INTRODUCTION}

An important challenge when detecting stationary targets through walls using electromagnetic (EM) waves is to locate the targets in the presence of wall EM reflections, which are relatively strong compared to behind-the-wall target return [15]. Therefore, it is necessary to mitigate the wall reflections prior to revealing the target positions.

There are several approaches for removing wall EM reflections from a signal. By estimating the parameters of the front wall, Dehmollaian and Sarabandi [1] modeled the EM wall return, which was then subtracted from the received data. Yoon and Amin [2] used a spatial filtering method to remove the direct current component corresponding to the wall return. Tivive et al. [3] mitigated the wall clutter by projecting the full data onto the subspace orthogonal to the wall-return subspace, which was estimated using a singular value decomposition (SVD).

Compressive sensing (CS), introduced at the early part of this century [6], has emerged as a new framework for signal acquisition using reduced numbers of measurements. Recently, CS has been used in radar systems to efficiently reduce acquisition cost [7, 8]. Lagunas et al. [4] considered applying both a spatial filtering method and an SVD-based subspace projection method to mitigate the wall return in compressive measurements. However, both methods require each antenna in a synthetic aperture array (SAR) to obtain the measurements with the same subset of frequencies. In the general case, when different frequency measurements are observed at different antenna locations, one needs to recover the full frequency measurements for each available antenna (using sparse recovery algorithms such as $l_{1}$-relaxation methods and iterative greedy methods) in order to employ conventional wall mitigation methods [4]. When using the Fourier basis to recover the full data, the corresponding "DFT leakage" results in the dispersion of wall clutter and worse target detection. To overcome this shortcoming, Ahmad et al. [5] utilized a dictionary of multiband modulated Discrete Prolate Spheroidal Sequences (DPSS's) [9, 10] to efficiently represent the wall return. In a recent paper [11], Ahmad et al. cancelled the wall clutter directly in the compressive domain by applying an orthogonal projection matrix (which relates to a Fourier basis) to the measurement vectors. Danvenport et al. [12] have provided theoretical results as well as experiments to support such a general framework of compressive signal processing.

In this paper, we provide an alternative way to [5] to use a modulated DPSS basis to cancel the wall return in the compressive domain. Only involving a subspace projection operator, our proposed method is much easier to implement compared to the method in [5], which involves a sparse recovery method. Furthermore, we show how the modulated DPSS basis can be applied to help detect stationary targets after the wall clutter has been mitigated.

The outline of this paper is as follows. In Section II, the main problem is illustrated. Our main approaches to cancel the wall return and detect the targets are in Section III. Section IV presents some simulations to support our proposed methods. Finally, some conclusions are given in Section V.

\section{PROBLEM SETUP}

Let $[N]$ denote the set $\{0,1, \ldots, N-1\}$ for any $N \in \mathbb{N}$. Suppose measurements are obtained by an $M$-element linear array of transceivers. Each transceiver receives a steppedfrequency signal consisting of $N$ frequencies equispaced over the band $\left[f_{0}, f_{N-1}\right]$; that is

$$
f_{n}=f_{0}+n \Delta F, \quad n \in[N],
$$

where $\Delta F:=\frac{f_{N-1}-f_{0}}{N-1}$ is the frequency step size.

According to [5], we can model the wall return at the $m$-th antenna corresponding to the $n$-th frequency as

$$
\boldsymbol{r}_{m}^{w}[n]:=\sum_{l=0}^{L} \vartheta_{l} e^{-j 2 \pi f_{n} t_{l, m}}, \forall m \in[M], n \in[N]
$$


Here, $\vartheta_{0}$ is the complex reflectivity of the wall; $\vartheta_{l}, l \geq 1$ represents the complex reflectivity corresponding to the $l$-th wall reverberation and decreases with $l ; L$ denotes the number of wall reverberations; $t_{0, m}$ is the direct two-way travel time between the wall and the $m$-th antenna; and $t_{l, m}, l \geq 1$ is the delay associated with the $l$-th wall return to the $m$-th antenna. Note that $t_{l, m}$ are the same for all $m \in[M]$ when the sensor array is parallel to the wall, which is a requirement in [4].

Suppose there are $K$ targets behind the wall. The target return observed by the $m$-th antenna corresponding to the $n$ th frequency can be expressed as

$$
\boldsymbol{r}_{m}^{t}[n]:=\sum_{k=1}^{K} \int_{\tau_{k, m}^{\min }}^{\tau_{k, m}^{\max }} \sigma_{k} e^{-j 2 \pi f_{n} \tau} d \tau, \forall m \in[M], n \in[N]
$$

Here, $\sigma_{k}$ is the complex reflectivity of the $k$-th target (we assume the target reflectivity is independent of frequency), and $\tau_{k, m}^{\min }$ and $\tau_{k, m}^{\max }$ are the minimum and maximum two-way travel times between the $k$-th target and the $m$-th antenna, respectively. Note that, when we model each target as a point, the target return degenerates to

$$
\boldsymbol{r}_{m}^{t}[n]=\sum_{k=1}^{K} \sigma_{k} e^{-j 2 \pi f_{n} \tau_{k, m}}
$$

where $\tau_{k, m}$ is the two-way travel time between the $k$-th point target and the $m$-th antenna [5].

The measurement $\boldsymbol{y}_{m}$ received by $m$-th antenna is the sum of $\boldsymbol{r}_{m}^{w}$ and $\boldsymbol{r}_{m}^{t}$; that is

$$
\boldsymbol{y}_{m}:=\boldsymbol{r}_{m}^{w}+\boldsymbol{r}_{m}^{t}
$$

for all $m \in[M]$.

In order to decrease the data size, CS techniques can be utilized to reduce the number of measurements $[4,5,11]$. Let $\Gamma \subset[M]$ denote the indices of the (randomly) selected antennas for collecting data, and assume $M_{1}:=\#(\Gamma)$ antennas are used. Let $\boldsymbol{\Phi}_{m}$ denote an $N_{1} \times N$ (often random) sensing matrix which is used at the $m$-th antenna to further reduce the sensing cost. A sensing matrix $\boldsymbol{\Phi}_{m}$ widely used for target detection applications is generated by randomly selecting $N_{1}$ rows from the $N \times N$ identity matrix $[4,5,11]$. In this case, computing $\boldsymbol{\Phi}_{m} \boldsymbol{y}_{m}$ simply involves picking $N_{1}$ entries from $\boldsymbol{y}_{m}$. We adopt this sensing strategy in this paper. Thus, our goal is to detect or localize the potential targets from compressive measurements $\left\{\boldsymbol{z}_{m}:=\boldsymbol{\Phi}_{m} \boldsymbol{y}_{m}\right\}_{m \in \Gamma}$.

\section{OUR APPROACH}

\section{A. A bandpass modulated DPSS basis}

The DPSS's are the bandlimited discrete-time sequences whose energies are most concentrated in a given time support [9]. Because we are primarily dealing with finite-length vectors in this paper, it is useful to consider the DPSS vectors, which can be obtained by restricting the DPSS's to their entries on the time indices $\{0,1, \ldots, N-1\}$. Let $\mathcal{I}_{N}$ denote the index-limiting operator that restricts a sequence to its entries on $\{0,1 \ldots, N-1\}$ (and produces a vector of length $N$ ), $\mathcal{I}_{N}^{*}$ be the adjoint operator of $\mathcal{I}_{N}$, and $\mathcal{B}_{W}$ represent a bandlimiting operator that bandlimits the DiscreteTime Fourier Transform (DTFT) of a discrete-time signal to the frequency range $[-W, W]$ (and returns the corresponding signal in the time domain). Given $N$ and $W \in\left(0, \frac{1}{2}\right)$, the DPSS vectors $s_{N, W}^{(0)}, s_{N, W}^{(1)}, \ldots, s_{N, W}^{(N-1)}$ (which form an orthobasis for $\mathbb{C}^{N}$ ) along with the corresponding eigenvalues $1>\lambda_{N, W}^{(0)}>\lambda_{N, W}^{(1)}>\cdots>\lambda_{N, W}^{(N-1)}>0$, satisfy $\mathcal{I}_{N}\left(\mathcal{B}_{W}\left(\mathcal{I}_{N}^{*}\left(\boldsymbol{s}_{N, W}^{(\kappa)}\right)\right)\right)=\lambda_{N, W}^{(\kappa)} \boldsymbol{s}_{N, W}^{(\kappa)}$ for all $\kappa \in[N]$.

The eigenvalues $\lambda_{N, W}^{(0)}, \lambda_{N, W}^{(1)}, \ldots, \lambda_{N, W}^{(N-1)}$ have a strong concentration behavior: the first $2 N W$ eigenvalues are close to 1 , while the remaining eigenvalues are close to 0 . This behavior enables us to use the first slightly more than $2 N W$ modulated DPSS vectors to represent sampled bandpass signals [9, 10]. Define $e_{f}:=\left[\begin{array}{llll}e^{j 2 \pi f 0} & e^{j 2 \pi f 1} & \cdots & e^{j 2 \pi f(N-1)}\end{array}\right]^{T}$ for all $f \in$ $\left[-\frac{1}{2}, \frac{1}{2}\right)$ as the sampled exponentials, where $T$ represents the transpose operator. Let $\boldsymbol{E}_{f_{c}}:=\operatorname{diag}\left(\boldsymbol{e}_{f_{c}}\right)$ denote an $N \times N$ diagonal matrix for any $f_{c} \in\left[-\frac{1}{2}, \frac{1}{2}\right)$. Also let $\boldsymbol{S}_{N, W}$ denote an $N \times N$ matrix that consists of all the DPSS vectors of length $N$ and bandwidth $W$, that is

$$
\boldsymbol{S}_{N, W}:=\left[\begin{array}{llll}
\boldsymbol{s}_{N, W}^{(0)} & \boldsymbol{s}_{N, W}^{(1)} & \cdots & \boldsymbol{s}_{N, W}^{(N-1)}
\end{array}\right] .
$$

Now define $\boldsymbol{Q}:=\left[\boldsymbol{E}_{f_{c}} \boldsymbol{S}_{N, W}\right]_{J}$ to be the first $J$ modulated DPSS vectors for some value of $J \in\{1,2, \ldots, N\}$ that we can choose as desired. The columns of $Q$ are orthonormal. Let $\boldsymbol{P}_{\boldsymbol{Q}}:=\boldsymbol{I}_{N}-\boldsymbol{Q} \boldsymbol{Q}^{H}$ denote an orthogonal projection from $\mathbb{C}^{N}$ to the orthogonal complement of the subspace formed by the columns of $\boldsymbol{Q}$. It is shown in [10] that when $J \approx 2 N W$ the dictionary $Q$ provides very accurate approximations (in an MSE sense) for finite-length sample vectors arising from sampling random bandpass signals.

Theorem III.1. ([10] Theorem 4.2.) Suppose $x(t)$ is a continuous-time, zero-mean, wide sense stationary random process with power spectrum

$$
P_{x}(F)= \begin{cases}\frac{1}{B_{\text {band }}}, & F \in\left[F_{c}-\frac{B_{\text {band }}}{2}, F_{c}+\frac{B_{\text {band }}}{2}\right], \\ 0, & \text { otherwise. }\end{cases}
$$

Let $\boldsymbol{x}=\left[\begin{array}{lll}x(0) & x\left(T_{s}\right) \ldots x\left((N-1) T_{s}\right)\end{array}\right]^{T} \in \mathbb{C}^{N}$ denote a finite vector of samples acquired from $x(t)$ with a sampling interval of $T_{s} \leq 1 /\left(2 \max \left\{\left|F_{c} \pm \frac{B_{\text {band }}}{2}\right|\right\}\right)$. Define $\boldsymbol{Q}:=\left[\boldsymbol{E}_{f_{c}} \boldsymbol{S}_{N, W}\right]_{J}$, where $f_{c}=F_{c} T_{s}$ and $W=\frac{B_{\text {band }} T_{s}}{2}$. For fixed $\epsilon \in(0,1)$, choose $J=2 N W(1+\epsilon)$. Then there exist constants $C_{1}, C_{2}, C_{3}, C_{4}$ and an integer $N_{0}$ (where $C_{2}, C_{4}, N_{0}$ may depend on $W, \epsilon$ ) such that

$$
\begin{aligned}
\mathbb{E}\left[\left\|\boldsymbol{P}_{\boldsymbol{Q}} \boldsymbol{x}\right\|_{2}^{2}\right] & =\frac{1}{2 W} \int_{f_{c}-W}^{f_{c}+W}\left\|\boldsymbol{P}_{\boldsymbol{Q}} \boldsymbol{e}_{f}\right\|_{2}^{2} d f \\
& \leq N \min \left(\epsilon+C_{1} e^{-C_{2} N}, \frac{C_{3}}{2 W} e^{-C_{4} N}\right),
\end{aligned}
$$

for all $N \geq N_{0}$. For comparison, $\mathbb{E}\left[\|\boldsymbol{x}\|_{2}^{2}\right]=\left\|\boldsymbol{e}_{f}\right\|_{2}^{2}=N$.

In fact we are also guaranteed such accuracy uniformly over all sampled sinusoids in the targeted bands by the following result.

Theorem III.2. With the same setup as in Theorem III.1, there exist constants $C_{5}, C_{6}$ (where $C_{5}, C_{6}$ may depend on $W$ and $\epsilon)$ such that

$$
\left\|\boldsymbol{P}_{\boldsymbol{Q}} \boldsymbol{e}_{f}\right\|_{2}^{2} \leq C_{5} N^{5 / 2} e^{-C_{6} N}, \forall f \in\left[f_{c}-W, f_{c}+W\right]
$$

for all $N \geq N_{0}$. 
Proof. We refer the reader to [13] due to limited space.

\section{B. Wall clutter mitigation}

Suppose the wall clutter $\boldsymbol{r}_{m}^{w}$ is well-approximated by a dictionary $\boldsymbol{D}_{m}$, which we discuss below. We cancel the wall return by constructing an operator $\boldsymbol{P}_{\left(\boldsymbol{\Phi}_{m} \boldsymbol{D}_{m}\right)}$ that operates on the measurement vector $\boldsymbol{z}_{m}$.

Ignoring the wall reverberations, the wall return defined in (1) reduces to $\boldsymbol{r}_{m}^{w}[n]:=\vartheta_{0} e^{-j 2 \pi f_{n} t_{0, m}}$. In this case, we can construct $\boldsymbol{D}_{m}$ as a vector with entries $\boldsymbol{D}_{m}[n]=$ $e^{-j 2 \pi f_{n} t_{0, m}}, \forall n \in[N]$, which was used in [11]. However, because of the wall reverberations, such a dictionary may not be able to represent the wall return well.

Suppose the parameters $t_{L, m}$ defined in (1) are known or can be estimated by knowing the wall material and building geometry. Define

$$
\boldsymbol{D}_{m}:=\left[\boldsymbol{E}_{-t_{0, m} \Delta F} \boldsymbol{S}_{N,\left(t_{L, m}-t_{0, m}\right) \Delta F}\right]_{J_{m}}
$$

to be the first $J_{m}$ modulated (by frequency $-t_{0, m} \Delta F$ ) DPSS vectors of length $N$ and bandwidth $\left(t_{L, m}-t_{0, m}\right) \Delta F$ with some value of $J_{m} \in\{1,2, \ldots, N\}$ for all $m \in \Gamma$.

We can rewrite the wall return in (1) as

$$
\boldsymbol{r}_{m}^{w}[n]=\sum_{l=0}^{L} \vartheta_{l} e^{-j 2 \pi f_{0} t_{l, m}} e^{-j 2 \pi n t_{l, m} \Delta F} .
$$

By noting that the wall return is a finite-length sample vector arising from sampling a bandpass signal in the band $\left[-t_{L, m} \Delta F,-t_{0, m} \Delta F\right]$, from Theorems III.1 and III.2, we expect that the wall return will be well-approximated by the dictionary $\boldsymbol{D}_{m}$.

Before moving on, we note that different bandpass modulated DPSS dictionaries could be constructed using different DPSS bandwidths and modulating frequencies from those suggested in (8). For example, one could also employ a dictionary such as $\boldsymbol{D}_{m}=\left[\boldsymbol{E}_{-} \frac{t_{0, m}+t_{L, m}}{2} \Delta F=\boldsymbol{S}_{N, \frac{\left(t_{L, m}-t_{0, m}\right)}{2} \Delta F}\right]_{J_{m}}$ to mitigate the wall return modeled in (1). However, EM simulations show that there also exists antenna ringing before the wall, which in addition to the wall reverberations can affect the detection of targets [5]. In consideration of the possible antenna ringing, we choose the bandpass modulated DPSS dictionary defined in (8).

It is also important to note that $t_{L, m}$ is directly determined by the wall material, whose electrical properties may not be known in advance. The dictionary $\boldsymbol{D}_{m}$ may also capture the energy in the target return located far behind the wall if $t_{L, m}$ is chosen to be too large, whereas a value of $t_{L, m}$ too small may result in a dictionary $\boldsymbol{D}_{m}$ that cannot capture the wall return completely. Simulations have shown that almost all walls have dominant reverberations up to $1.5 \mathrm{~m}$ behind the wall [5]. Therefore, we use the same strategy as [5] in that we mitigate the wall reverberations up to $1.5 \mathrm{~m}$ behind the wall and detect only the targets located more than $1.5 \mathrm{~m}$ beyond the wall. That is, the basis defined in (8) turns out to be

$$
\boldsymbol{D}_{m}:=\left[\boldsymbol{E}_{-t_{0, m} \Delta F} \boldsymbol{S}_{N, \frac{3}{c} \Delta F}\right]_{J_{m}}
$$

where the constant $c$ is the speed of light in meters per second.
We know $\boldsymbol{r}_{m}^{w}$ is approximately within the column space of $\boldsymbol{D}_{m}$. It follows that $\boldsymbol{\Phi}_{m} \boldsymbol{r}_{m}^{w}$ is mostly concentrated in the column space of $\boldsymbol{\Phi}_{m} \boldsymbol{D}_{m}$. Now define an orthogonal projection matrix $\boldsymbol{P}_{\left(\boldsymbol{\Phi}_{m} \boldsymbol{D}_{m}\right)}:=\boldsymbol{I}_{N_{1}}-\boldsymbol{\Phi}_{m} \boldsymbol{D}_{m}\left(\boldsymbol{\Phi}_{m} \boldsymbol{D}_{m}\right)^{\dagger}$, where $\boldsymbol{I}_{N_{1}}$ is an $N_{1} \times N_{1}$ identity matrix and $\dagger$ denotes the pseudo-inverse operator. Applying $\boldsymbol{P}_{\boldsymbol{\Phi}_{m} \boldsymbol{D}_{m}}$ to the signal $\boldsymbol{z}_{m}$ yields

$\widetilde{\boldsymbol{z}}_{m}:=\boldsymbol{P}_{\left(\boldsymbol{\Phi}_{m} \boldsymbol{D}_{m}\right)} \boldsymbol{z}_{m}=\boldsymbol{P}_{\left(\boldsymbol{\Phi}_{m} \boldsymbol{D}_{m}\right)} \boldsymbol{\Phi}_{m} \boldsymbol{r}_{m}^{t}+\boldsymbol{P}_{\left(\boldsymbol{\Phi}_{m} \boldsymbol{D}_{m}\right)} \boldsymbol{\Phi}_{m} \boldsymbol{r}_{m}^{w}$. Since $\boldsymbol{P}_{\left(\boldsymbol{\Phi}_{m} \boldsymbol{D}_{m}\right)} \boldsymbol{\Phi}_{m} \boldsymbol{r}_{m}^{w} \approx \mathbf{0}$, we get $\widetilde{\boldsymbol{z}}_{m} \approx \boldsymbol{P}_{\left(\boldsymbol{\Phi}_{m} \boldsymbol{D}_{m}\right)} \boldsymbol{\Phi}_{m} \boldsymbol{r}_{m}^{t}$.

Now the projected measurements $\left\{\widetilde{\boldsymbol{z}}_{m}\right\}_{m \in \Gamma}$ can be used to detect the targets.

\section{Target detection}

Like the general approach for radar imaging [5], we divide the target space into $L_{x} \times L_{y}$ pixels in crossrange and downrange uniformly. We arrange the pixels of the image into an $L_{x} L_{y} \times 1$ vector $\boldsymbol{\alpha}$. If we were to assume the targets were points and located precisely on the grid, then using the point target model in (3), the target return could be written as

$$
\boldsymbol{r}_{m}^{t}=\boldsymbol{\Theta}_{m} \boldsymbol{\alpha}, \forall m \in \Gamma,
$$

where $\boldsymbol{\Theta}_{m}$ is an $N \times L_{x} L_{y}$ matrix with entries given by $\boldsymbol{\Theta}_{m}[n, q]:=e^{-j 2 \pi f_{n} \tau_{q, m}}$ for $n \in[N]$ and $q \in$ $\left\{0,1, \ldots, L_{x} L_{y}-1\right\}$. Here $\tau_{q, m}$ denotes the two-way travel time between the $q$-th potential target position in the grid and the $m$-th transceiver. Note that in this model $\boldsymbol{\alpha}$ is a sparse vector: the value of $q$-th pixel equals the target reflectivity if there is a target at this pixel, and otherwise it is 0 . Therefore,

$$
\widetilde{\boldsymbol{z}}_{m} \approx \boldsymbol{P}_{\left(\boldsymbol{\Phi}_{m} \boldsymbol{D}_{m}\right)} \boldsymbol{\Phi}_{m} \boldsymbol{\Theta}_{m} \boldsymbol{\alpha}=: \widetilde{\boldsymbol{\Theta}}_{m} \boldsymbol{\alpha} .
$$

The processed measurements $\left\{\widetilde{\boldsymbol{z}}_{m}\right\}_{m \in \Gamma}$ are arranged into an $M_{1} N_{1} \times 1$ vector $\widetilde{z}$ as

$$
\widetilde{\boldsymbol{z}}=\left[\begin{array}{llll}
\widetilde{\boldsymbol{z}}_{m_{0}}^{H} & \widetilde{\boldsymbol{z}}_{m_{1}}^{H} & \ldots & \widetilde{\boldsymbol{z}}_{m_{M_{1}-1}}^{H}
\end{array}\right]^{H},
$$

where $m_{0}, m_{1}, \ldots, m_{M_{1}-1} \in \Gamma$ and $H$ represents the conjugate transpose. The measurements can be expressed as

$$
\widetilde{z} \approx \Theta \alpha,
$$

where

$$
\boldsymbol{\Theta}:=\left[\begin{array}{llll}
\widetilde{\boldsymbol{\Theta}}_{m_{0}}^{H} & \widetilde{\boldsymbol{\Theta}}_{m_{1}}^{H} & \cdots & \widetilde{\boldsymbol{\Theta}}_{m_{M_{1}-1}}^{H}
\end{array}\right]^{H} .
$$

It is known that we can recover the sparse vector $\alpha$ from the projected measurements $\widetilde{z}$ provided that the matrix $\Theta$ satisfies certain properties.

However, if the target does not fall precisely on the grid, or in the more general case, if the target is not a point and the target return is as defined in (2), then $\boldsymbol{\alpha}$ will not be sparse. Especially when the dynamic range of the target reflectivities is large, then the target return corresponding to the largest target reflectivity may dominate and sparse solvers may fail to detect the other targets.

In order to detect and localize the targets in this general case, we modify the iterative, greedy matching pursuit (MP) algorithm [14] (which is identical to the CLEAN algorithm [15]) so that the energy of exponentials with two-way traveling time within $\varsigma$ seconds to that of each selected point is cancelled by using a modulated DPSS basis. For each 
$q \in\left\{0,1, \ldots, L_{x} L_{y}-1\right\}$ and $m \in \Gamma$, define a compressive bandpass modulated DPSS basis as

$$
\boldsymbol{\Psi}_{q, m}:=\boldsymbol{\Phi}_{m}\left[\boldsymbol{E}_{-\tau_{q, m} \Delta F} \boldsymbol{S}_{N, \Delta F \varsigma}\right]_{J^{t}}
$$

with $J^{t}$ slightly larger than $2 N \Delta F \varsigma$. Next define the orthogonal projection matrix

$$
\boldsymbol{P}_{\Psi_{q}}:=\left[\begin{array}{lll}
\boldsymbol{P}_{\boldsymbol{\Psi}_{q, m_{0}}} & & \\
& \ddots & \\
& & \boldsymbol{P}_{\boldsymbol{\Psi}_{q, m_{M}-1}}
\end{array}\right],
$$

where $\boldsymbol{P}_{\boldsymbol{\Psi}_{q, m_{\iota}}}:=\boldsymbol{I}_{N_{1}}-\boldsymbol{P}_{\boldsymbol{\Phi}_{m_{\iota}} \boldsymbol{D}_{m_{\iota}}} \boldsymbol{\Psi}_{q, m_{\iota}}\left(\boldsymbol{P}_{\mathbf{\Phi}_{m_{\iota}} \boldsymbol{D}_{m_{\iota}}} \boldsymbol{\Psi}_{q, m_{\iota}}\right)^{\dagger}$ for all $\iota \in\left[M_{1}\right]$. By applying $\boldsymbol{P}_{\boldsymbol{\Psi}_{q}}$ to the vector $\widetilde{\boldsymbol{z}}$, and by judiciously choosing $\varsigma$ (which we discuss below), we can remove essentially all the target return corresponding to the spatial region near grid point $q$. This is the main difference between our proposed algorithm and the conventional MP algorithm. The full DPSS-aided MP algorithm for target detection is shown in Algorithm 1.

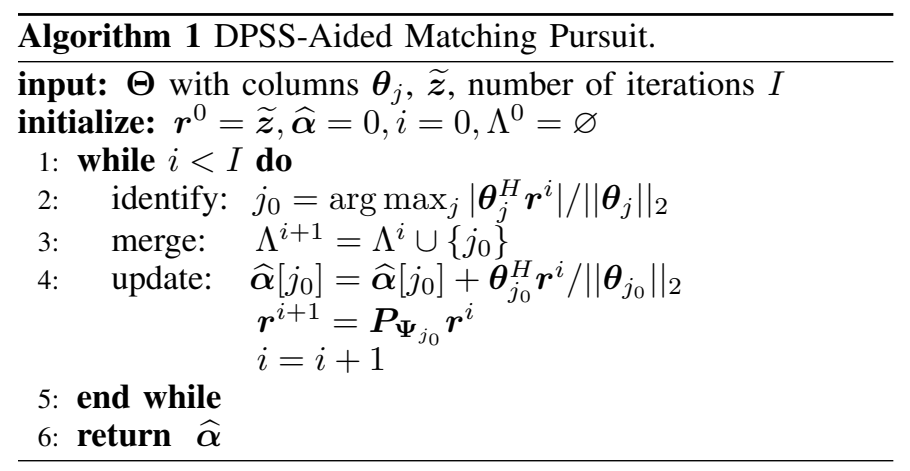

\section{Simulations}

With a similar scene layout to that in [5], we simulate three $0.35 \mathrm{~m} \times 0.28 \mathrm{~m}$ targets located at $(-0.29 \mathrm{~m}, 5.38 \mathrm{~m})$, $(1.55 \mathrm{~m}, 6.38 \mathrm{~m})$, and $(-1.69 \mathrm{~m}, 6.53 \mathrm{~m})$, with relative complex reflectivities of 5,1 , and 1 , respectively. A 93-element synthetic linear aperture (located along the $x$-axis) with interelement spacing of $0.04 \mathrm{~m}$ is used. A stepped-frequency signal consisting of $N=641$ frequencies from $1 \mathrm{GHz}$ to $4 \mathrm{GHz}$ is utilized to obtain measurements. We simulate with only a front wall, which is located $3.13 \mathrm{~m}$ away from the antennas. The scene layout is illustrated in Fig. 1(a). $30 \%$ of the antennas are randomly selected to acquire measurements. We then randomly choose $30 \%$ of the frequencies for each available antenna.

The $4 \mathrm{~m} \times 5.5 \mathrm{~m}$ region centered at $(0 \mathrm{~m}, 4.75 \mathrm{~m})$ is chosen to be imaged, and it is divided into a grid of $33 \times 77$ pixels. The target return is generated according to (2). On the basis of (1), we generate $L=5$ wall reverberations equally spaced between the wall and $1.5 \mathrm{~m}$ behind the wall with $\vartheta_{0}=30$ and $\vartheta_{l}=\frac{1}{1+l} \vartheta_{0}$ for all $l=1, \ldots, L$.

The number $J_{m}$ for the bandpass modulated DPSS dictionary $\boldsymbol{D}_{m}$ is chosen to be 70 . We construct $\boldsymbol{\Psi}_{q, m}$ defined in (16) with $\varsigma=2 \mathrm{~ns}$ and $J^{t}=20$ so that we can cancel the target return corresponding to the area with two-way travel time within $2 \mathrm{~ns}$ to that of any selected point.

We compare our wall mitigation method using a bandpass modulated DPSS basis to the one using a Fourier basis in [11] and the one based on a multiband modulated DPSS dictionary in [5]. The construction of the multiband modulated DPSS dictionary is the same as that used in [5], which includes choosing $5.5 \mathrm{~ns} \cdot \Delta F$ as the bandwidth for the DPSS vectors.

Figures 2(a-c) show the sparsity-based target image reconstruction with 15 iterations of the OMP algorithm (assuming target sparsity in a Fourier basis of complex exponentials), whereas Fig. 2(d) displays the target reconstruction result with 3 iterations of the DPSS-aided matching pursuit algorithm. Fig. 2(e) shows the average result over 50 trials of the DPSSaided matching pursuit algorithm. A Fourier basis used in [11], a multiband modulated DPSS dictionary used in [5], and a bandpass modulated DPSS dictionary are applied for mitigating the wall return in Figs. 2(a), (b) and (c-d), respectively.

Comparing the bottom region in Fig. 2(a) to those in Figs. 2(b-d), we find that the DPSS-based dictionary can capture the wall return better than the Fourier basis. Both the multiband modulated DPSS dictionary and the bandpass modulated DPSS dictionary can be used to mitigate the wall return well. However, the bandpass modulated DPSS dictionary is much easier to implement compared to the multiband modulated DPSS dictionary, which involves a sparse recovery method such as orthogonal matching pursuit (OMP, which was used in our simulation for Fig 2(b)) in [5]. Clearly, we observe in Fig. 2 (d) that the proposed DPSS-aided MP algorithm can find the top two targets, while the Fourier-based algorithm misses these targets in Figs. 2(b-c). Note that the proposed algorithm will miss the shape of targets. There is a tradeoff between identifying the shapes of the targets and identifying their locations. By changing $\varsigma$, we can balance this tradeoff depending on the particular application.

We point out that a modified Fourier-domain OMP algorithm may also be able to offer improved target detection. To be precise, we modify OMP so that in each iteration when we pick one pixel in the grid, we also choose its neighbors. For example, when the red point in Fig. 1(b) is selected, the nearby 48 black points are chosen along with the red one. This subFourier basis corresponding to the 49 points can be viewed as a surrogate basis for the subspace spanned by sampled bandpass signals in this local band. This is closely related to the main modification of our proposed DPSS-aided MP algorithm, in which a bandpass modulated DPSS basis is used to represent the sampled bandpass signals. Fig. 3 shows the singular values of a sub-Fourier basis corresponding to the 49 points (where the red point is randomly picked), which indicates that the effective dimension of this sub-Fourier basis is smaller than 49. The target reconstruction result is shown in Fig. 2(e).

\section{CONCLUSIONS}

In this paper, we have explained how a modulated DPSS basis can help in mitigating wall return and detecting stationary targets after the wall clutter has been cancelled. This is possible thanks to the remarkable efficiency of the bandpass modulated DPSS dictionary in representing finite-length sample vectors of bandpass signals. Experiments show the effectiveness of the modulated DPSS basis in cancelling the wall return and promoting target detection.

\section{ACKNOWLEDGMENT}

This work was supported by NSF grant CCF-1409261. 


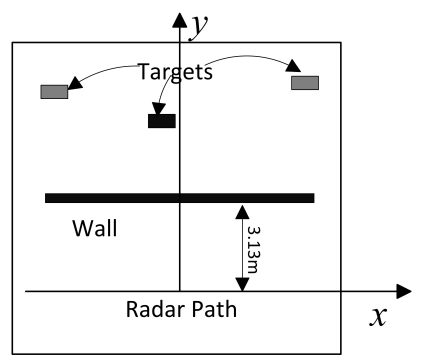

(a)

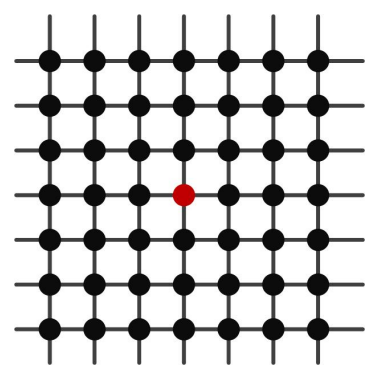

(b)
Fig. 1. Illustration of (a) scene layout, and (b) point selection strategy used in modified Fourier-domain OMP algorithm

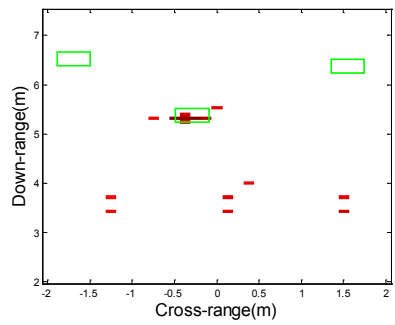

(a)

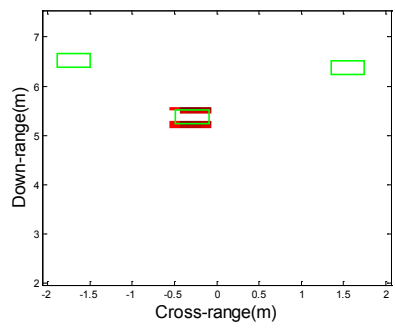

(c)

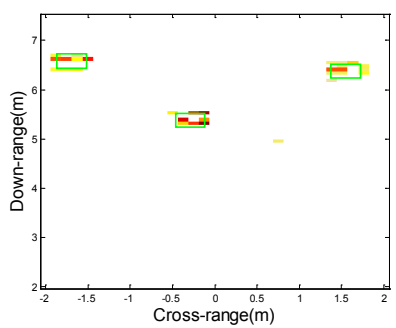

(e)

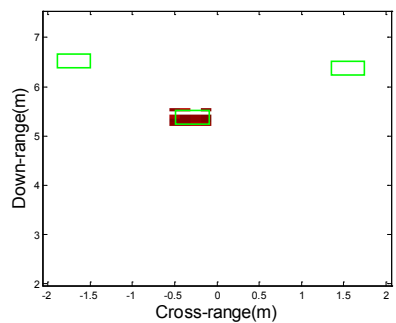

(b)

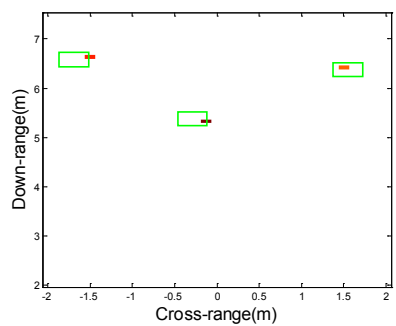

(d)

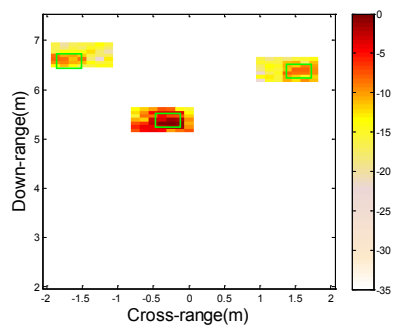

(f)
Fig. 2. Wall mitigation and sparse reconstruction results with different methods (the maximum intensity value is normalized to $0 \mathrm{~dB}$ ). (a) Wall mitigation with Fourier basis and sparse reconstruction using OMP algorithm with Fourier basis. (b) Wall mitigation with multiband modulated DPSS dictionary and sparse reconstruction using OMP algorithm with Fourier basis. (c) Wall mitigation with bandpass modulated DPSS basis and sparse reconstruction using OMP algorithm with Fourier basis. (d) Wall mitigation with bandpass modulated DPSS basis and sparse reconstruction using DPSS-aided MP algorithm. (e) Average result over 50 trials for method presented in (d). (f) Wall mitigation with bandpass modulated DPSS basis and sparse reconstruction using modified Fourier-domain OMP algorithm with 3 iterations.

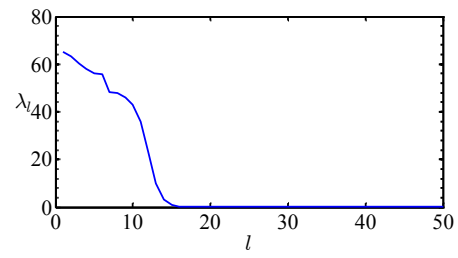

Fig. 3. Singular values of a sub-Fourier basis constructed from 49 sampled complex exponentials.

\section{REFERENCES}

[1] M. Dehmollaian and K. Sarabandi, "Refocusing through building walls using synthetic aperture radar," IEEE Trans. Geosci. Remote Sens., vol. 46, pp. 1589-1599, June 2008.

[2] Y.-S. Yoon and M. Amin, "Spatial filtering for wallclutter mitigation in through-the-wall radar imaging," IEEE Trans. Geosci. Remote Sens., vol. 47, pp. 31923208, Sept 2009.

[3] F. Tivive, A. Bouzerdoum, and M. Amin, "An SVDbased approach for mitigating wall reflections in throughthe-wall radar imaging," in Proc. 2011 IEEE Radar Conference, pp. 519-524, May 2011.

[4] E. Lagunas, M. Amin, F. Ahmad, and M. Najar, "Joint wall mitigation and compressive sensing for indoor image reconstruction," IEEE Trans. Geosci. Remote Sens., vol. 51, pp. 891-906, Feb 2013.

[5] F. Ahmad, J. Qian, and M. Amin, "Wall clutter mitigation using Discrete Prolate Spheroidal Sequences for sparse reconstruction of indoor stationary scenes," IEEE Trans. Geosci. Remote Sens., vol. 53, pp. 1549-1557, March 2015.

[6] E. Candès and M. Wakin, "An introduction to compressive sampling," IEEE Signal Process. Mag., vol. 25, pp. 21-30, March 2008.

[7] R. Baraniuk and P. Steeghs, "Compressive radar imaging," in Proc. 2007 IEEE Radar Conference, pp. 128133, April 2007.

[8] M. Amin, Compressive sensing for urban radar. CRC Press, 2014.

[9] D. Slepian, "Prolate Spheroidal Wave Functions, Fourier analysis, and uncertainty-V: The discrete case," Bell Syst. Tech. J, vol. 57, no. 5, pp. 1371-1430, 1978.

[10] M. A. Davenport and M. B. Wakin, "Compressive sensing of analog signals using Discrete Prolate Spheroidal Sequences," Appl. Comput. Harmon. Anal., vol. 33, no. 3, pp. 438-472, 2012.

[11] F. Ahmad, M. Amin, and T. Dogaru, "Partially sparse imaging of stationary indoor scenes," EURASIP J. Adv. Signal Process., vol. 2014, no. 1, pp. 1-15, 2014.

[12] M. Davenport, P. Boufounos, M. Wakin, and R. Baraniuk, "Signal processing with compressive measurements," IEEE. J. Sel. Topics in Signal Process., vol. 4, pp. 445460, April 2010.

[13] Z. Zhu and M. B. Wakin, "New analysis of multiband modulated DPSS dictionaries," in preparation.

[14] S. G. Mallat and Z. Zhang, "Matching pursuits with timefrequency dictionaries," IEEE Trans. Signal Process., vol. 41, no. 12, pp. 3397-3415, 1993.

[15] J. Tsao and B. D. Steinberg, "Reduction of sidelobe and speckle artifacts in microwave imaging: The CLEAN technique," IEEE Trans. Antenn. Propag., vol. 36, no. 4, pp. 543-556, 1988. 